\title{
Early science with MeerKAT
}

\section{Sharmila Goedhart*}

South African Radio Astronomy Observatory, 2 Fir Street, Black River Park, Observatory, 7925,

Cape Town, South Africa

E-mail: sharmila@ska.ac.za

The MeerKAT telescope celebrated its inauguration on 13 July 2018. While it is still being commissioned, and more correlator capabilities are expected to be rolled out, it has already demonstrated its potential to produce high dynamic range images with high fidelity, as shown with its inaugural image of the Galactic Centre. A technical overview of MeerKAT, its measured performance, current status and future capabilities is provided and first light images are shown.

High Energy Astrophysics in Southern Africa - HEASA2018

1-3 August, 2018

Parys, Free State, South Africa

${ }^{*}$ Speaker. 


\begin{tabular}{|c|c|}
\hline Number of antennas & 64 \\
\hline slew speed & $2 \mathrm{deg} / \mathrm{s}$ \\
\hline Antenna diameter & $13.5 \mathrm{~m}$ \\
\hline mimimum baseline & $29 \mathrm{~m}$ \\
\hline maximum baseline & $7.7 \mathrm{~km}$ \\
\hline lower elevation limit & $15 \mathrm{deg}$ \\
\hline field of view at $1.4 \mathrm{GHz}$ & $0.85 \mathrm{deg}^{2}$ \\
\hline field of view at $0.58 \mathrm{GHz}$ & $5 \mathrm{deg}^{2}$ \\
\hline
\end{tabular}

Table 1: Antenna and array specifications

\section{Introduction}

The MeerKAT telescope is an interferometric radio telescope array consisting of 64 dishes in the Northern Cape near Carnarvon, South Africa. It is a precursor for the mid-frequency Square Kilometer Array (SKA) telescope and a scientific pathfinder for the SKA. MeerKAT is a general purpose telescope that will operate at UHF, L and $S$ bands. Prior to finalising the MeerKAT design, a call for large survey proposals (more than 1000 hours) was made in 2009 [1]. Ten projects were approved, which were used to refine the MeerKAT user requirements specification. Broad scientific areas include pulsar search and timing, transient detection and monitoring, deep $\mathrm{HI}$ and continuum surveys. Details of MeerKAT science cases can be found in the proceedings of MeerKAT Science: On the pathway to SKA [2].

Construction of the full array was completed in March 2018, and the telescope was inaugurated on 13 July 2018. Commissioning and science verification of the full array commenced at this time, and is still ongoing at time of writing, while new correlator capabilities are being developed and additional receiver bands are being installed. At this stage the telescope is operating in the the so-called wideband coarse continuum imaging mode in L-band.

Section 2 will provide the telescope specification and measured performance. Section 3 will briefly summarise the MeerKAT large survey projects and a few early results will be discussed in section 4.

\section{Telescope specification}

Detailed descriptions of the design constraints and implementation of the MeerKAT telescope is given in [3] and [4]. We present here the basic telescope characteristics and specifications that may be of interest to potential users.

The antenna and array characteristics are summarised in Table 1. The MeerKAT antennas have a 'feed-down' offset Gregorian configuration (Figure 1) which creates an unblocked aperture with efficiency of 0.71 to 0.81 across UHF to L-band.

The MeerKAT array configuration is optimised to have a compact core for pular observing and imaging extended low-surface brightness sources, while still providing moderate angular resolution (up to 6" at L-band). The baseline spacing provides uniform sensitivity for angular scales ranging from $8^{\prime \prime}$ to $80 "$ at $1420 \mathrm{MHz}$. 


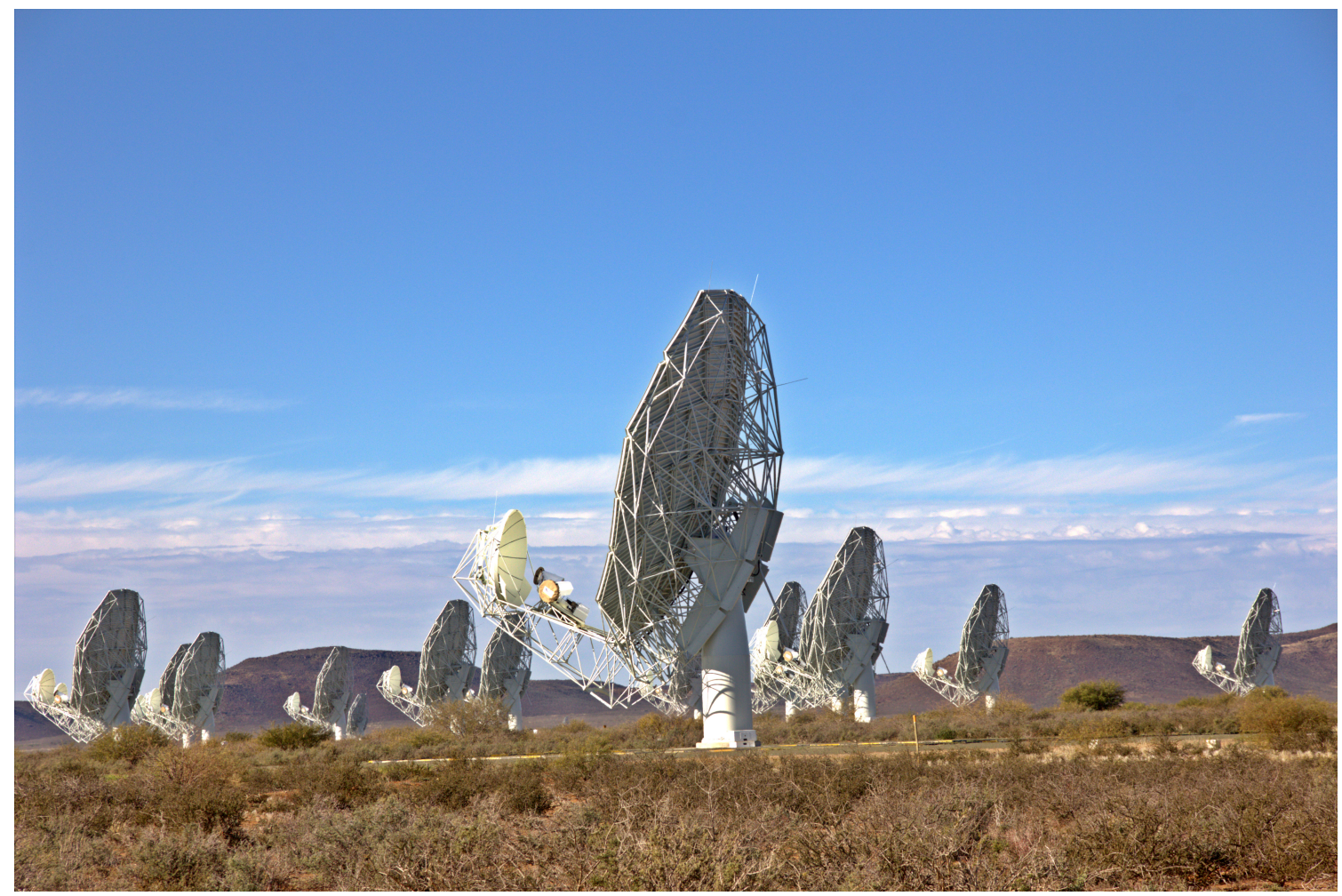

Figure 1: Antennas in the core of the MeerKAT array.

\begin{tabular}{|l|l|l|l|}
\hline Band & $\begin{array}{l}\text { Frequency range } \\
(\mathrm{GHz})\end{array}$ & $\begin{array}{l}\text { digitised bandwidth } \\
(\mathrm{MHz})\end{array}$ & status \\
\hline L & $0.9-1.67$ & 856 & operational \\
\hline UHF & $0.58-1.015$ & 544 & installing \\
\hline S & $1.75-3.5$ & 875 & prototype testing \\
\hline
\end{tabular}

Table 2: MeerKAT frequency bands and status

MeerKAT will be equiped with three receiver bands (Table 2) on a rotating carousel, with provision made to mount a fourth receiver. The L-band receiver systems are fully functional and in regular use for commissioning, science verification and early science. The UHF receivers have been installed, while installation of all UHF digitisers is expected to be completed in the first half of 2019, after which commissioning and science verification tests will commence. The S-band receiver system is being provided by the MPIfR [5]. Two prototypes are currently being tested on the array.

The correlator/beamformer (CBF) is based on a reconfigurable architecture [6] to enable operation in different modes tailored to specific science cases. Table 3 lists early modes to be deployed. Full polarisation correlation products as well as auto-correlations are always recorded. Further modes and data products may be available as the platform matures and new requirements emerge. The wideband coarse mode is well-characterised and being used for continuum imaging. The wideband fine mode is in an early integration and testing phase while the narrowband modes are still in 


\begin{tabular}{|c|c|c|c|c|}
\hline Mode & $\begin{array}{c}\text { channelised band } \\
\text { (MHz) }\end{array}$ & channels & $\begin{array}{c}\text { channel width } \\
(\mathrm{kHz})\end{array}$ & $\begin{array}{c}\text { velocity resolution } \\
(\mathrm{km} / \mathrm{s})\end{array}$ \\
\hline wideband coarse (4K) & 856 & 4096 & 209 & 44.6 \\
\hline wideband fine (32K) & 856 & 32768 & 26 & 5.5 \\
\hline narrowband extended & $108,56,25$, or 13 & 32768 & 0.4 to 3.3 & 0.08 to 0.7 \\
\hline
\end{tabular}

Table 3: Correlator modes and spectral resolution at L-band, $1.4 \mathrm{GHz}$

development. A beamformer mode with 1024 channels and a single boresight beam has also been developed for pulsar timing and searches.

Commissioning tasks are proceeding well at L-band and include:

- Antenna pointing calibration: Blind pointing models good to $\approx 2^{\prime}$ have been derived. The long term stability of the pointing solutions is still under investigation and referenced pointing methods (necessary for high dynamic range wide-field imaging) are being developed.

- Antenna position and delay models: Baseline calibration tests using unresolved point sources with well known positions have converged to within $3 \mathrm{~mm}$ on antenna positions at L-band.

- Signal chain gain: Optimal attenuation settings for the L-band digitisers have been established. Beamformer, X-engine and F-engine gains as well as FFT-shift schedules have to be determined for each correlator mode.

- Stability of calibration solutions: Antenna phase solutions show a standard deviation of 2.5 deg over a 20 minute rolling window. The spectral baseline is stable to $\approx-25 \mathrm{~dB}$ level over 8 hours.

Further commissioning and science verification involve observation of a wide range of targets to establish stability, sensitivity and dynamic range. Sensitive observations were made of a field selected to be free of bright sources (to avoid dynamic range limitations). A 16-hour track of this field achieved an rms noise of $1.87 \mu \mathrm{Jy} / \mathrm{beam}$ (T. Mauch, priv. comm.). Mosaicing and imaging of extended structures has also been demonstrated, as will be shown in section 4 .

Figure 2 shows the typical single-dish system-equivalent flux density (SEFD) spectrum at Lband for the horizontal and vertical polarisation channels, measured by driftscans over Tau A. The greyscale shows areas of persistent RFI, however this tends to decorrelate on longer baselines. Optimisation of signal chain gain and shift settings is expected to further mitigate the effects of RFI.

\section{Key science programme}

Ten large survey projects were approved in 2010. Large survey projects are allocated twothirds of science observing time, $30 \%$ of time will be allocated through competitive open time calls and approximately 5\% of time may allocated at the director's discretion (DDT).

The following eight projects will be using the UHF and/or L-band receivers. 


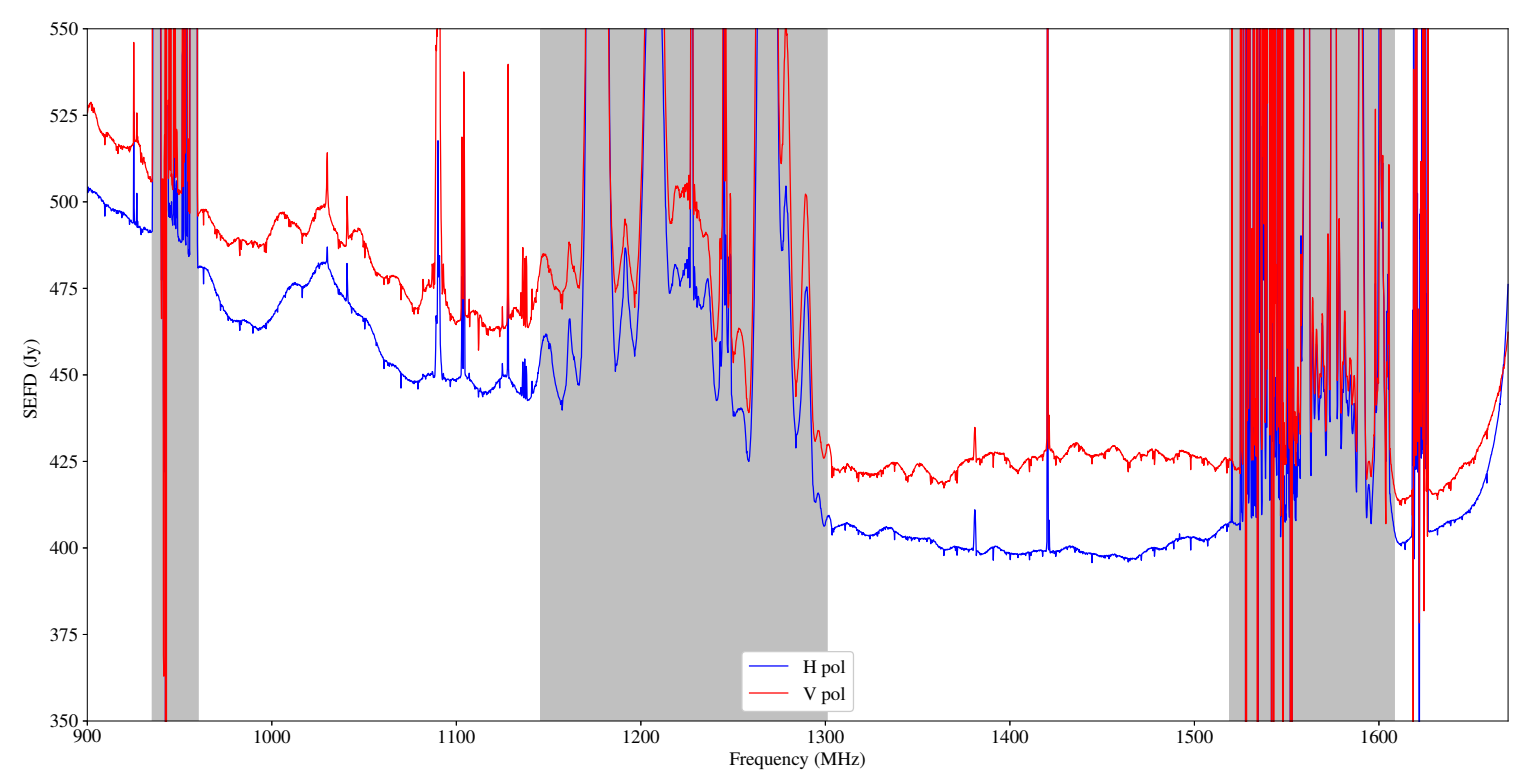

Figure 2: The measured SEFD of a typical MeerKAT antenna at L-band. Masked areas are heavily and persistently contaminated by radio frequency interference.

- LADUMA - Looking at the distant universe with the MeerKAT Array - is an ultradeep HI survey out to a redshift of 1.4. This survey will use both UHF and L-band to cover the full redshift range. Start of observations depends on integration and commissioning of the wideband fine correlator mode.

- MALS - MeerKAT absorption line survey - is a survey of HI and $\mathrm{OH}$ absorbers out to a redshift of 2, using both $\mathrm{L}$ and UHF bands. Start of observations depends on integration and commissioning of wideband fine correlator mode.

- The MeerKAT Fornax Survey is a deep HI emission survey of the Fornax cluster. Start of observations depends on integration and commissioning of narrowband extended correlator mode.

- MeerTIME will conduct timing of different classes of pulsars. First observations were successfully completed in February 2019.

- MHONGOOSE - MeerKAT HI Observations of Nearby Galactic Objects: Observing Southern Emitters - will map the neutral hydrogen in a sample of 30 nearby galaxies. Start of observations depends on integration and commissioning of narrowband extended correlator mode.

- MIGHTEE - The MeerKAT International GHz Tuned Extragalactic Exploration Survey is a deep extragalactic continuum survey. First pathfinder observations were executed in 2018. Start of full survey depends on integration and commissioning of wideband fine correlator mode to maximise commmensal observations. 
- ThunderKAT - The Hunt for Dynamic and Explosive Radio Transients with MeerKAT - will monitor high energy transient sources associated with accretion and explosive events such as supernovae. Regular observations are being scheduled.

- TRAPUM - Tansients and Pulsars with MeerKAT - will conduct targeted and blind surveys of fast transients and pulsars. Start of observations depends on integration and commissioning of user-supplied equipment.

Two additional surveys, MESMER (a high redshift CO survey) and MeerGAL (a $14 \mathrm{GHz}$ survey of the Galactic Plane) proposed to use the X-band receiver specified in the original call for proposal. However, funding and time constraints make the implementation of this band uncertain.

\section{Early Science}

\subsection{Array Release 1 (AR1)}

The AR1 correlator was based upon an earlier iteration of the correlator hardware amd could only process dual polarisation inputs from 16 antennas. Two target of opportunity projects led to the first MeerKAT publications while AR1 was still being commissioned.

In March 2017, the magnetar PSR J1622-4950 reactivated and radio emission was detected by the Parkes telescope on 5 April 2017. While the beamformer mode was not fully integrated and commissioned yet, MeerKAT was in a unique position to be able to monitor this object while Parkes underwent a planned month-long shutdown in May 2017 [4]. Timing solutions from MeerKAT observations were used to provide ephemerides for Chandra and NuStar, and to monitor the shortterm variability of the spin-down torque on the neutron star.

On 17 August 2017, LIGO and VIRGO detected gravitational waves generated by a potential binary neutron star merger (GW170817). MeerKAT participated in the multi-messenger campaign to monitor the resulting transient source found near the galaxy NGC 4993 [7]. The first observations were made with 12 antennas between the 25th to 27th August 2017. Unfortunately the maximum baseline available was $4.87 \mathrm{~km}$ and the image was dominated by artefacts generated by a strong source situated across the first null of the primary beam. At this time MeerKAT could only report a 3-sigma upper detection limit of $60 \mu \mathrm{Jy}$ at $1.3 \mathrm{GHz}$. Monitoring of this source continued, while advanced calibration methods to mitigate the effects of the bright interfering source were investigated. MeerKAT, in its AR1 configuration successfully detected the radio source at its peak of $160 \mu \mathrm{Jy}$ in March 2018 and subsequently at $65.8 \mu \mathrm{Jy}$ in April 2018 using the full 64-element array [8]. Monitoring continues and a follow-up publication is in preparation.

An open call for early shared-risk science with AR1 was issued in February 2018. This covered both continuum and spectral line imaging. 14 proposals were received and 12 were observed prior to the decommissioning of the AR1 correlator in May 2018. Some 156 hours of telescope time were allocated and 15.8 TB of data was recorded. Data reduction and analysis by the investigators is currently under way.

\subsection{MeerKAT-64}

The full MeerKAT array achieved its first fringes on 29 March 2018. The first commissioning observations were conducted in April 2018 and gave such promising results that an ambitious 
series of observations were planned for the inauguration. Figure 3 shows a $1.3^{\circ} \times 1^{\circ}$ section of the Galactic plane. This is part of a six-pointing mosaic that was observed in a single night. The MeerKAT measurements are overlaid on the Spitzer GLIMPSE mid-infrared image. Supernova remnants G332.4+01 (asymmetrical bubble to top left), G332.0+0.2 (symmetrical faint bubble top right of centre) and G332.4-0.4 (bottom left of centre) can be seen. HII emission from massive star forming clusters can be seen lighting up the cavities shown in the Spitzer image, which is dominated by emission from dust. Close examination will reveal several background active galactic nuclei (AGN) as well.

The most notable image produced by MeerKAT to date is a mosaic of the Galactic centre (zoomed area of $2.17^{\circ} \times 1.137^{\circ}$ shown in Figure 4). The full mosaic consists of 26 ten-hour tracks of pointings on a hexagonal grid. This image is the sharpest and most sensitive image in L-band of the Galactic centre to date. MeerKAT's unique baseline configuration makes it ideally suited to image structures over a large range of angular scales. Analysis of this data is in progress and a publication is in preparation.

In the past year, while integration and commissioning of MeerKAT is the predominant activity, science observations are conducted during the nights and on weekends. These consist of limited observations for the Large Survey Projects, as outlined in section 3, and two observatory-led legacy projects: a survey of the fourth quadrant of the Galactic Plane, and a survey of galaxy clusters. These data are in the process of being verified, and will be released at a later stage.

\section{Summary and Outlook}

The MeerKAT telescope has commenced its science programme, while still undergoing commissioning. Additional capabilities are still being developed and commissioned to unlock its full scientific potential.

A call for open time proposals was released in December 2018. Proposals are currently under review. We look forward to many exciting discoveries with MeerKAT, and the continued growth of the South African radio astronomy community.

\section{References}

[1] Booth R.S., de Blok W.J.G., Jonas J.L., Fanaroff B. MeerKAT Key Project Science, Specifications, and Proposals, 2009, [arXiv: 0910.2935]

[2] Proceedings of MeerKAT Science: On the Pathway to the SKA. 25-27 May, 2016 Stellenbosch, South Africa Editors: A.R. Taylor, F. Camilo, L. Leeuw, K. Moodley [https://pos.sissa.it/277/]

[3] J.L. Jonas and MeerKAT Team, The MeerKAT Radio Telescope in proceedings of MeerKAT Science: On the Pathway to the SKA. 25-27 May, 2016 Stellenbosch, South Africa PoS (MeerKAT2016) 001 (2016)

[4] Camilo F., Scholz P., Serylak M., Buchner S., Merryfield M., Kaspi V. M., Archibald R. F., Bailes M., Jameson A., van Straten W. et al., Revival of the Magnetar PSR J1622-4950: Observations with MeerKAT, Parkes, XMM-Newton, Swift, Chandra, and NuSTAR, 2018, ApJ, 856, 180

[arXiv:1804.01933] 


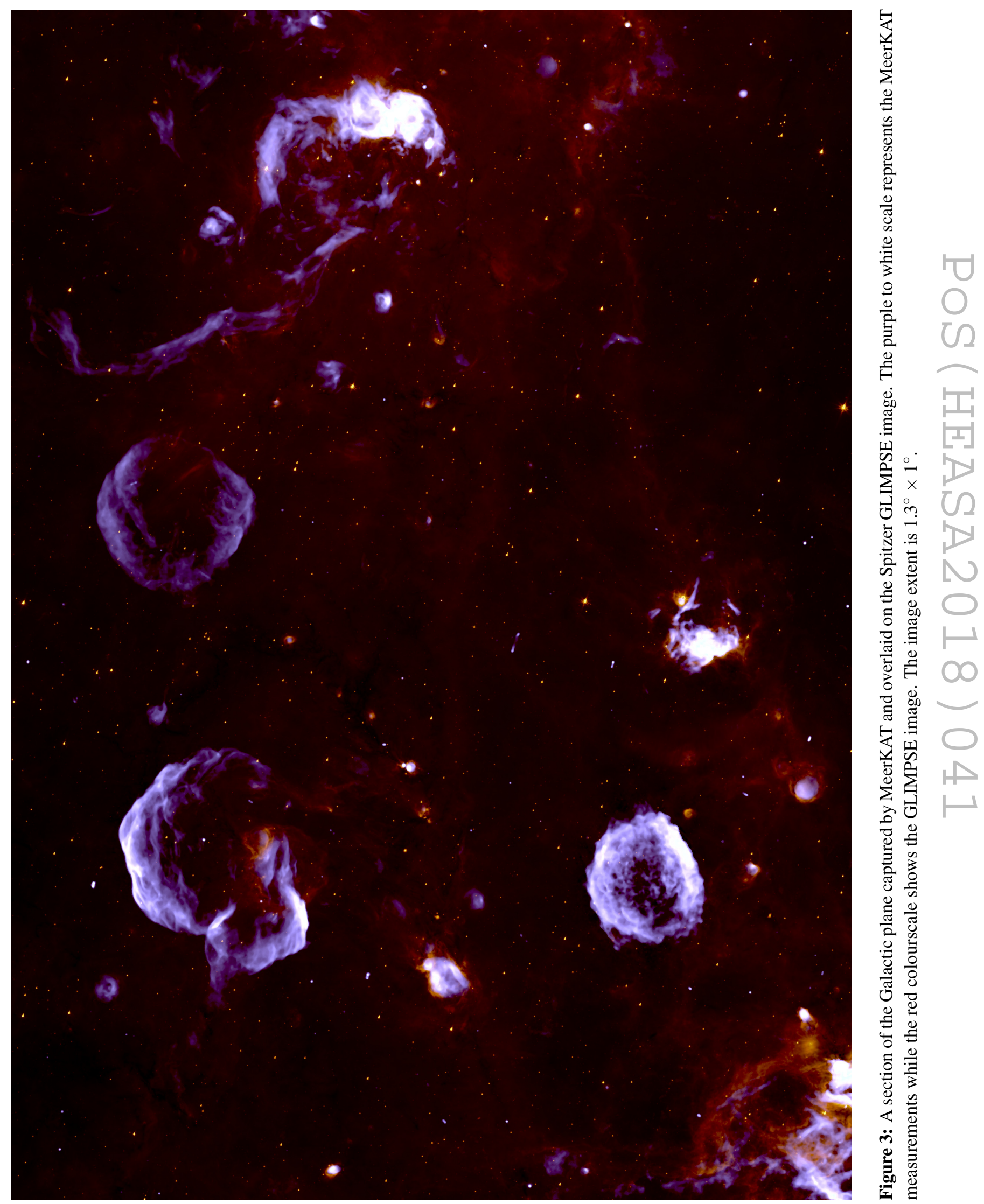




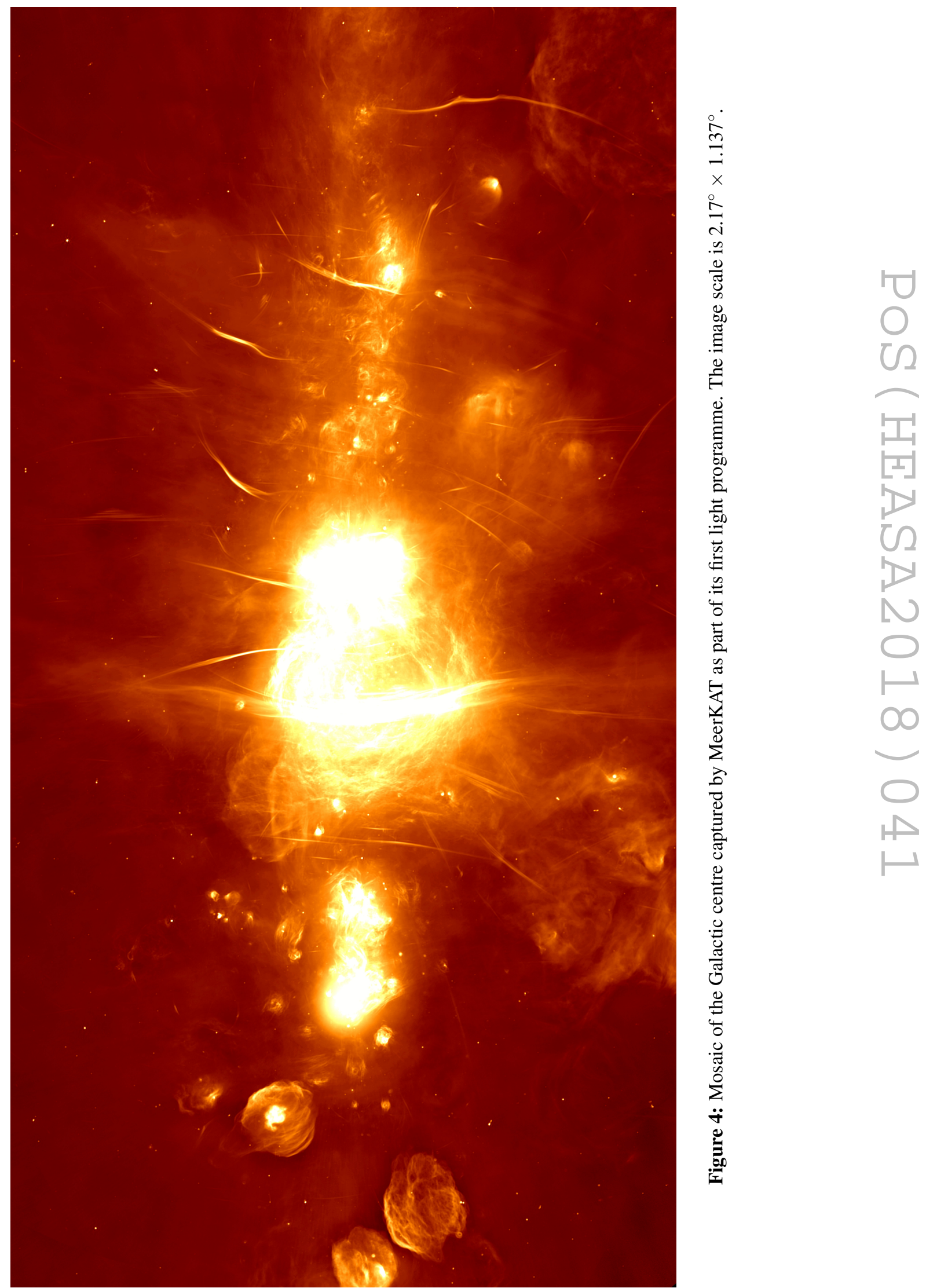


[5] M. Kramer, K. Menten, E.D. Barr, R. Karuppusamy, C. Kasemann, B. Klein, E. Ros, G. Wieching, O. Wucknitz, The MeerKAT Max-Planck S-band system, in proceedings of MeerKAT Science: On the Pathway to the SKA. 25-27 May, 2016 Stellenbosch, South Africa PoS (MeerKAT2 016 ) 003 (2016)

[6] Hickish J., Abdurashidova Z., Ali Z., Buch K. D., Chaudhari S. C, Chen H., Dexter M., Domagalski R. S., Ford J., Foster G., George D., Greenberg, J., Greenhill L., Isaacson A., Jiang H., Jones G., Kapp F., Kriel H., Lacasse R., Lutomirski A., MacMahon D., Manley J., Martens A., McCullough R., Muley M. V., New W., Parsons A., Price D. C., Primiani R. A., Ray J., Siemion A., van Tonder V., Vertatschitsch L., Wagner M., Weintroub J., Werthimer D., A Decade of Developing Radio-Astronomy Instrumentation using CASPER Open-Source Technology, 2016, JAI, Volume 5, Issue 4, id. 1641001-12, [arXiv:1611.01826]

[7] Abbott B. P., Abbott R., Abbott T. D., Acernese F., Ackley K., Adams C., Adams T., Addesso P., Adhikari R. X., Adya V. B., et al. Multi-messenger observations of a binary neutron star merger, 2017, ApJ, 848L, 12 [arXiv:1710.05833]

[8] Mooley K. P., Frail D. A., Dobie D., Lenc E., Corsi A., De K., Nayana A. J., Makhathini S., Heywood I., Murphy T., Kaplan D. L., Chandra P., Smirnov O., Nakar E., Hallinan G., Camilo F., Fender R., Goedhart S., Groot P., Kasliwal M. M., Kulkarni S. R., Woudt P. A. A Strong Jet Signature in the Late-time Light Curve of GW170817, 2018, ApJ, 868L, 11 [arXiv:1810.12927 ] 